\title{
Poincaré is a subgroup of Galilei in one space dimension more
}

\author{
Emili Elizalde ${ }^{a)}$ \\ Departament de Física Teòrica, Universitat de Barcelona, Diagonal 647, Barcelona-14. Spain.
}

\begin{abstract}
Through an imaginary change of coordinates, the ordinary Poincare algebra is shown to be a subalgebra of the Galilei one in four space dimensions. Through a subsequent contraction the remaining Lie generators are eliminated in a natural way. An application of these results to connect Galilean and relativistic field equations is discussed.
\end{abstract}

\section{INTRODUCTION}

Some papers have been issued ${ }^{1}$ which deal with the connection between the usual relativistic field equations (those of Dirac, Bargmann and Wigner, Proca, Rarita and Schwinger, and Singh and Hagen $)^{2}$ and the nonrelativistic ones (of Lévy-Leblond and Hagen and Hurley). ${ }^{3}$ In particular, for example, from the Dirac eqution one obtains that of Lévy-Leblond ${ }^{3}$ for a spin $-\frac{1}{2}$ particle, and subsequently the Schrödinger-Pauli equation, and starting with the Bargmann-Wigner equation for an arbitrary spin particle one obtains the $6 s+1$ Galilean invariant theory of Hagen and Hurley. ${ }^{3}$

These connections have been established by means of a general change of the coordinates of the Minkowski space, which has the property of showing up a Galilean $(2+1)$-dimensional Lie subalgebra in the ordinary Poincaré algebra. ${ }^{4}$ The usual light-cone frame ${ }^{2}$ and the nonorthogonal one of Bell and Ruegg ${ }^{5}$ are interesting particular cases of this general coordinate transformation. Moreover, making use of a convenient parametrization of this coordinate transformation a $(2+1)$ dimensional Poincaré algebra can be reobtained from the Galilean subalgebra in a continuous way and for a particular value of the parameter. Thus the circle is closed, making possible, in particular, the calculation of higher-order terms of the Schrödinger-Pauli equation derived before. ${ }^{4}$

It is the purpose of the present paper to go one step ahead of this program by studing in a more general manner the connection between the Galilei and Poincaré algebras. It will be shown by means of a certain imaginary coordinate transformation of the $(4+1)$ dimensional space-time frame-which changes the fourth spatial and the time coordinates-that the ordinary Poincare algebra is a subalgebra of that of Galilei in four space dimensions. With a subsequent contraction of the Lie group, the rest of the generators will be consistently eliminated, and what will remain are exactly the commutation relations of the ordinary Poincare group. We think that this procedure will be of much use in the derivation of relativistic field equations for any spin starting from Galilean invariant ones, in a process inverse to the one which has been employed before. ${ }^{1}$ Anyway, we are not going to develop these possibilities here, where we only concern ourselves with the mathematics of the problem.

\footnotetext{
a) Address from October 1977: II. Institut für Theoretische Physik der Universität Hamburg.
}

Finally, we want to point out that the results of the present paper confirm the feeling ${ }^{1,4}$ that the loss of one space dimension in the light-cone frame-and in the Galilean equations thereby obtained-is not of much relevance. In other words, it has been conjectured $^{1,4}$ that the correct ordinary nonrelativistic expressions in three space dimensions would be obtained in the light-cone frame provided one started with the corresponding relativistic ones in one more space coordinate. Although in the contrary direction, this is also proved in this work.

\section{FROM THE GALILEI TO THE POINCARE ALGEBRA}

Let $x^{\mu}=\left(x^{0}, x^{1}, x^{2}, x^{3}, x^{4}\right)$ denote a point in a $(4+1)$ dimensional space-time frame, $x^{0}$ being the time coordinate, and let the coordinates of the same point in a new frame $\bar{x}^{\mu}=\left(\bar{x}^{0}, \bar{x}^{1}, \bar{x}^{2}, \bar{x}^{3}, \bar{x}^{4}\right)$ be defined as

$$
\begin{aligned}
& \bar{x}^{0}=a x^{0}+b x^{4}, \\
& \bar{x}^{i}=x^{i} \quad(i=1,2,3), \\
& \bar{x}^{4}=c x^{0}+d x^{4},
\end{aligned}
$$

where $a, b, c$, and $d$ are some constants, to be determined in order that the commutation relations of the Lie algebra of the Galilei group in the old frame be transformed into those of the ordinary Poincaré group in the new system.

Before going on let us recall that the Galilei group $G$ in $4+1$ dimensions can be put into the for $\mathrm{m}^{6}$

$$
G=\left[\mathrm{SO}(4) \times T_{4}^{(v)}\right] \times\left[T_{1} \otimes T_{4}\right],
$$

where $T_{4}^{(v)}$ is the subgroup of the generators of Galilean boosts and $T_{4}$ that of the tranlations in 4-space. As is usual, $\times$ means semidirect and $\otimes$ direct product.

Now let

$$
\begin{aligned}
& x^{0}=A \vec{x}^{0}+B \bar{x}^{4}, \quad A=\frac{d}{a d-b c}, \quad B=\frac{-b}{a d-b c}, \\
& x^{4}=C \bar{x}^{0}+D \bar{x}^{4}, \quad C=\frac{-c}{a d-b c}, \quad D=\frac{a}{a d-b c}
\end{aligned}
$$

be the inverse transformation of $(2.1)$, and let $l_{i}, \lambda_{i}$ $(i=1,2,3)$ be the generators ${ }^{7}$ of the subgroup $\mathrm{SO}(4)$ of $G, g_{r}(r=1,2,3,4)$ those of the Galilean boosts, and $d_{\mu}(\mu=0,1,2,3,4)$ the generators of the timespace translations. If we denote with a bar the corresponding generators in the new coordinate system, we have 


$$
\begin{aligned}
& \bar{l}_{i}=l_{i}, \quad \bar{d}_{i}=d_{i}, \quad \bar{g}_{i}=A g_{i}-C \lambda_{i}, \\
& \bar{d}_{0}=A d_{0}+C d_{4} \quad(i=1,2,3) .
\end{aligned}
$$

As it has already been pointed out, ${ }^{4,8}$ the choice of the new generators of boosts (which we call $k_{i}$ for a reason that will become obvious in a moment) is not so clear. In general, let us put $k_{i}=\alpha g_{i}+\beta \lambda_{i}(i=1,2,3), \bar{g}_{i}$ being one of the possibilities, while the other two are $\bar{\lambda}_{i}$ (Bjorken, Kogut, and Soper) $)^{8}$ and $M^{0 i}$ (quasilight-cone frame $)^{4}$ where $M_{\mu \nu}$ are the generators of $\mathrm{SO}(4) \times T_{4}^{(v)}$.

The commutation relations of the generators of the Galilei group in $4+1$ dimensions are the following. In the first place, for the rotation group $\mathrm{SO}(4)$ we have

$$
\begin{gathered}
{\left[R_{r s}, R_{u v}\right]=} \\
i\left(\delta_{r v} R_{s u}+\delta_{s u} R_{r v}-\delta_{r u} R_{s v}-\delta_{s v} R_{r u}\right) \\
(r, s, u, v=1,2,3,4)
\end{gathered}
$$

or putting $l_{i}=-\frac{1}{2} \epsilon_{i j k} R_{j k}, \lambda_{i}=R_{4 i}(i, j, k=1,2,3)$, the equivalent ones

$$
\begin{aligned}
& M_{\mu \nu}=\left(\begin{array}{cccc}
0 & A g_{1}-C d_{1} & A g_{2}-C \lambda_{2} & A g_{3}-C \lambda_{3} \\
-A g_{1}+C \lambda_{1} & 0 & l_{3} & -l_{2} \\
-A g_{2}+C \lambda_{2} & -l_{3} & 0 & l_{1} \\
-A g_{3}+C \lambda_{3} & l_{2} & -l_{1} & 0 \\
-(A D-B C) g_{4} & B g_{1}-D \lambda_{1} & B g_{2}-D \lambda_{2} & B g_{3}-D \lambda_{3}
\end{array}\right. \\
& d_{\mu}=\left(A d_{0}+C d_{4}, d_{1}, d_{2}, d_{3}, B d_{0}+D d_{4}\right),
\end{aligned}
$$

and the possible choices for $k_{i}$ which we have mentioned above are the following:

$$
\begin{array}{ll}
k_{i}=\bar{g}_{i}=A g_{i}-C \lambda_{i} & \text { (natural), } \\
k_{i}=\bar{l}_{i}=-B g_{i}+D \lambda_{i} & \text { (light-cone frame), } \\
k_{i}=\bar{M}^{0 i}=-\left(a g_{i}+b \lambda_{i}\right) & \text { (quasi-light-cone frame), }
\end{array}
$$

It is easy to see that in the transformed frame, the commutation relations of the generators $l_{i}, k_{i}, d_{i}$ $(i=1,2,3)$, and $h \equiv d_{0}$, are given by

$\left[l_{i}, l_{j}\right]=i \epsilon_{i j k} l_{k}, \quad\left[l_{i}, k_{j}\right]=i \epsilon_{i j k} k_{k},\left[l_{i}, d_{j}\right]=i \epsilon_{i j k} d_{k}$,

$\left[k_{i}, k_{j}\right]=i \beta^{2} \epsilon_{i j k} l_{k}, \quad\left[d_{i}, d_{j}\right]=\left[l_{i}, h\right]=\left[d_{i}, h\right]=0$

$\left[k_{i}, h\right]=i(\alpha A-\beta C) d_{i},\left[k_{i}, d_{j}\right]=i\left(x \mu+\beta b h+\beta d \bar{d}_{4}\right) \delta_{i j}$.

These equalities constitute the Lie algebra of the ordinary Poincaré group, provided we put

$$
\alpha=0, \quad \beta^{2}=-1, \quad \beta b=1, \quad \beta d=0, \quad \alpha A-\beta C=1
$$

or, equivalently,

$$
\alpha=0, \quad \beta= \pm i, \quad b=\mp i, \quad d=0, \quad C= \pm i .
$$

The first choice of $k_{i}$ is consistent with these conditions when $A=0$ and $C= \pm i, B$ and $D$ remaining arbitrary. In this case $k_{i}=\mp i \lambda_{i}(i=1,2,3)$. Also the second choice

$$
\begin{aligned}
& {\left[l_{i}, l_{j}\right]=i \epsilon_{i j k} l_{k},} \\
& {\left[l_{i}, \lambda_{j}\right]=i \epsilon_{i j k} \lambda_{k} \quad(i, j, k=1,2,3),} \\
& {\left[\lambda_{i}, \lambda_{j}\right]=i \epsilon_{i j k} l_{k} .}
\end{aligned}
$$

Moreover,

$\left[\lambda_{i}, g_{j}\right]=i \delta_{i j} g_{4}, \quad\left[\lambda_{i}, d_{j}\right]=i \delta_{i j} d_{4}, \quad\left[\lambda_{i}, d_{0}\right]=0$,

$\left[\lambda_{i}, g_{4}\right]=-i g_{i}, \quad\left[\lambda_{i}, d_{4}\right]=-i d_{i}$,

$\left[l_{i}, g_{4}\right]=\left[l_{i}, d_{4}\right]=0 \quad(i, j=1,2,3)$.

And finally, the Lie algebra of the ordinary Galilei group,

$\left[l_{i}, l_{j}\right]=i \epsilon_{i j k} l_{k}, \quad\left[l_{i}, g_{j}\right]=i \epsilon_{i j k} g_{k}, \quad\left[l_{i}, d_{j}\right]=i \epsilon_{i j k} d_{k}$

$\left[g_{i}, g_{j}\right]=\left[d_{i}, d_{j}\right]=\left[l_{i}, d_{0}\right]=\left[d_{i}, d_{0}\right]=0, \quad\left[g_{i}, d_{0}\right]=i d_{i}$,

$\left[g_{i}, d_{j}\right]=i \delta_{i j} \mu, \quad\left[l_{i}, \mu\right]=\left[d_{i}, \mu\right]=\left[g_{i}, \mu\right]=\left[d_{0}, \mu\right]=0$

in a true eleven-parameter group representation.

In Eqs. (2.5) - (2.7) we have listed the whole set of commutation relations of our Galilei group in the original frame. After the coordinate transformation has been made, the new set of generators is given by

$$
\left.\begin{array}{c}
(A D-B C) g_{4} \\
-B g_{1}+D \lambda_{1} \\
-B g_{2}+D \lambda_{2} \\
-B g_{3}+D \lambda_{3} \\
0
\end{array}\right),
$$

is consistent, taking $A=0, B$ arbitrary, $C=D= \pm i$, and $k_{i}= \pm i \lambda_{i}$. Finally, for the third choice we have $a=d=0, b= \pm i, c$ arbitrary, and $k_{i}=\mp i \lambda_{i}$. This is the one we are going to study in more detail. ${ }^{4}$

Summing up, through the change of coordinates

$$
\begin{array}{ll}
\bar{x}^{0}= \pm i x^{4}, & x^{0}=(1 / c) \bar{x}^{4}, \\
\vec{x}^{i}=x^{i} \quad(i=1,2,3) & x^{i}=\bar{x}^{i}, \\
\bar{x}^{4}=c x^{0} \quad(c \text { arbitrary }) & x^{4}=\mp i \overrightarrow{x^{0}},
\end{array}
$$

the commutation relations for the transformed generators $l_{i}, k_{i}, d_{i}(i=1,2,3)$, and $h$, are those of the ordinary Poincare algebra. The rest of the transformed generators are easily seen to be given by

$$
\bar{\lambda}_{i}=-(1 / c) g_{i}, \quad \bar{g}_{4}=(i / c) g_{4}, \quad \bar{d}_{4}=(1 / c) d_{n}
$$

and their commutation relations by

$\left[\bar{\lambda}_{i}, \bar{\lambda}_{j}\right]=0, \quad\left[l_{i}, \bar{\lambda}_{j}\right]=i \epsilon_{i j k} \bar{\lambda}_{k}, \quad\left[\bar{\lambda}_{i}, k_{j}\right]=-i \delta_{i j} g_{4}$,

$\left[\bar{\lambda}_{i}, g_{4}\right]=0, \quad\left[\bar{\lambda}_{i}, d_{j}\right]=-(i / c) \delta_{i j} \mu, \quad\left[\bar{\lambda}_{i}, h\right]=0$,

$\left[\bar{\lambda}_{i}, \bar{d}_{4}\right]=-(i / c) d_{i}$,

$\left[l_{i}, \bar{g}_{4}\right]=0,\left[\bar{k}_{i}, \overline{g_{4}}\right]=i \lambda_{i}, \quad\left[\overline{g_{4}}, d_{i}\right]=0, \quad\left[\overline{g_{4}}, h\right]=(i / c) \mu$, $\left[\bar{g}_{4}, \bar{d}_{4}\right]=\left(i / c^{2}\right) h_{2},\left[l_{i}, \bar{d}_{4}\right]=\left[k_{i}, \bar{d}_{4}\right]=\left[d_{i}, \bar{d}_{4}\right]=\left[h, \bar{d}_{4}\right]=0$. 
Notice that when $c \rightarrow \infty \bar{d}_{4}$ becomes a neutral element of the transformed Lie algebra. On the other hand, as the value of $c$ is arbitrary we can make it go to infinity and, then, the only commutation relations of (2.15) which remain different from zero are the following:

$\left[l_{i}, \bar{\lambda}_{j}\right]=i \epsilon_{i j k} \bar{\lambda}_{k}, \quad\left[\bar{\lambda}_{i}, k_{j}\right]=-i \delta_{i j} \bar{g}_{4}, \quad\left[k_{i}, \bar{g}_{4}\right]=i \bar{\lambda}_{i}$.

At the same time, observe that in (2.14) we also can make $c$ as large as we like and, in this way, the generators $\bar{\lambda}_{i}, \bar{g}_{4}$, and $\bar{d}_{4}$ become negligible.

\section{CONCLUSIONS}

Starting with the Galilei group in four space/one time dimensions and making the imaginary change of coordinates given by (2.13), we have seen that the commutation relations satisfied by the generators transformed of $l_{i}, g_{i}, d_{i}(i=1,2,3)$, and $d_{0}$ are exactly those of the ordinary Poincare algebra in the Minkowski space. That the transfor mation must be imaginary is clear if we notice that the Euclidean matrix $\delta_{\mu \nu}$ must be converted into the Lorentz's $g_{\mu \nu}$. Mor eover, the transformed of the other five generators can be made as little as we like without affecting in the least the commutation relations which define the Poincare algebra. Therefore, the transformation which has been carried out here can be defined as an imaginary change of coordinates followed by a contraction of the resulting Lie algebra with respect to the subalgebra of the generators $(2.10)$ which satisfy the Poincaré relations.

Following a procedure parallel to the one developed elsewhere, ${ }^{1,4}$ we presume that this result may be of much use in relating Galilean field equations with relativistic invariant ones and, particularly, to obtain the latter from the former, in just the reciprocal way to the one employed till now. The contraction of one space dimension which takes place in the light-cone frame, ${ }^{2,8}$ i.e., the ordinary relativistic equations in the Minkowski space give rise in this frame to Galilean invariant ones in $2+1$ dimensions, also occurs here. In order to prove that this contraction does not depend on the particular number $3+1$ of dimensions of the Minkowski space, we have started here with a $(4+1)$-dimensional Galilei algebra. As expected, the spatial contraction has carried us to a $(3+1)$-dimensional Poincaré world. Extrapolating this procedure to an arbitrary number $n$ of space dimensions, it is plausible to believe that the method develped here would transform a $(n+1)$-dimensional Galilei algebra into a $[(n-1)+1]$-dimensional Poincaré one while, at the same time, it looks appealing to think that the light-cone frame procedure would lead from a $(n+1)$-dimensional Poincaré algebra to a Galilei one in $(n-1)+1$ dimensions. Naturally this will have its parallel counterpart at the level of the wave equations, which always appear in the number of space-time dimensions of the corresponding Lie group.

Let us finish by saying that the ultimate purpose of this paper has been to find new and deeper connections between the mathematical structure of relativistic and Galilean Lie groups, connections which we hope will be useful in order to throw some light into the rather complicated world of their corresponding field equations for different spin particles.

${ }^{1} \mathrm{E}$. Elizalde and J. Gomis, Nuovo Cimento A $35,336,347$, 367 (1976).

${ }^{2}$ L.P.S. Singh and C.R. Hagen, Phys. Rev. D 9, 898,910 (1974)。

${ }^{3}{ }^{3}$. -M. Lévy-Leblond, Comm. Math. Phys. 6, 286 (1967); C.R. Hagen and W. J. Hurley, Phys. Rev. Lett. 24, 1381 (1970); C. R. Hagen, Comm. Math. Phys, 18, 97 (1970) ; W. J. Hurley, Phys. Rev. D 3, 2339 (1971).

${ }^{4}$ E. Elizalde and J. Gomis, Nucl. Phys. B 122, 535 (1977). ${ }^{5}$ J. S. Bell and H. Ruegg, Nucl. Phys。B 93, $12(1976)$.

${ }^{6} \mathrm{E}$. G. Sudarshan and N. Mukunda, Classical Dynamics (Wiley, New York, 1974).

${ }^{7} \mathrm{H}$. Bacry, Leçons sur la thêorie des groupes et les symétries des particules elémentaires (Gordon and Breach, Paris, 1967). See also original references there.

${ }^{8}$ J. B. Kogut and D. E. Soper, Phys. Rev. D 1, 2901 (1970); J.D. Bjorken, J. B. Kogut, and D. E. Soper, Phys. Rev. D 3 , $1382(1971)$. 